\title{
A prospective multicenter phase II study evaluating multimodality treatment of patients with peritoneal carcinomatosis arising from appendiceal and colorectal cancer: the COMBATAC trial
}

Gabriel Glockzin ${ }^{1 *}$, Justine Rochon ${ }^{2}$, Dirk Arnold ${ }^{3}$, Sven A Lang ${ }^{1}$, Frank Klebl ${ }^{4}$, Florian Zeman ${ }^{5}$, Michael Koller ${ }^{5}$, Hans J Schlitt ${ }^{1}$ and Pompiliu Piso ${ }^{1,6}$

\begin{abstract}
Background: Peritoneal carcinomatosis is regarded as a common sign of advanced tumor stage, tumor progression or local recurrence of appendiceal and colorectal cancer and is generally associated with poor prognosis. Although survival of patients with advanced stage CRC has markedly improved over the last 20 years with systemic treatment, comprising combination chemotherapy $+/$ - monoclonal antibodies, the oncological outcome-especially of the subgroup of patients with peritoneal metastases - is still unsatisfactory. In addition to systemic therapy, cytoreductive surgery (CRS) and hyperthermic intraperitoneal chemotherapy (HIPEC) are specific treatment options for a selected group of these patients and may provide an additional therapeutic benefit in the framework of an interdisciplinary treatment concept.

Methods/design: The COMBATAC trial is a prospective, multicenter, open-label, single-arm, single-stage phase II trial investigating perioperative systemic polychemotherapy including cetuximab in combination with CRS and HIPEC patients with histologically proven wild-type KRAS colorectal or appendiceal adenocarcinoma and synchronous or metachronous peritoneal carcinomatosis. The planned total number of patients to be recruited is 60 . The primary endpoint is progression-free survival (PFS). Secondary endpoints include overall survival (OS), perioperative morbidity and treatment-associated toxicity, feasibility of the combined treatment regimen, quality of life (QoL) and histopathological regression after preoperative chemotherapy.

Discussion: The COMBATAC trial is designed to evaluate the feasibility and efficacy of the combined multidisciplinary treatment regimen consisting of perioperative systemic combination chemotherapy plus cetuximab and CRS plus bidirectional HIPEC with intraperitoneal oxaliplatin.

Trial registration: ClinicalTrials.gov Identifier: NCT01540344, EudraCT number: 2009-014040-11

Keywords: Cytoreductive surgery, HIPEC, Perioperative chemotherapy, Cetuximab, Colorectal cancer, Peritoneal carcinomatosis
\end{abstract}

\footnotetext{
* Correspondence: gabriel.glockzin@ukr.de

'Department of Surgery, University Medical Center Regensburg, Regensburg 93042, Germany

Full list of author information is available at the end of the article
} 


\section{Background}

\section{Disease under study}

Colorectal cancer (CRC) is the third most commonly diagnosed cancer in males and the second in female worldwide and overall the fourth leading cause of cancer-related death. Whereas the mortality associated with CRC slightly decreased over the past 20 years the incidence is still increasing in most countries [1,2]. More than $10 \%$ of patients with CRC already show peritoneal carcinomatosis at the time of initial diagnosis [3]. In about $25 \%$ of the cases there is no evidence of further distant metastasis [4]. Moreover, up to $25 \%$ of all patients with CRC develop peritoneal carcinomatosis during the natural course of their disease as a common sign of tumor progression or recurrence. In contrast to lymphatic and hematologic spread of metastases, intraperitoneal carcinomatosis develops by direct transcolonic tumor spread or tumor cell seeding during surgical resection of the primary tumor [5-8]. Tumor cell distribution within the abdominal cavity results in avascular tumor nodules that often cannot be efficiently addressed by systemic chemotherapy [9]. Thus, peritoneal carcinomatosis is mostly associated with poor prognosis. In the prospective European multicenter EVOCAPE 1 study, a median survival of 5.2 months was reported out of the 118 patients with peritoneal carcinomatosis arising from CRC during the natural course of disease [10]. Another retrospective analysis of 3,000 patients with peritoneal colon cancer dissemination reported a comparable median survival of 7 months [11].

\section{First-line treatment of advanced colorectal cancer}

Systemic chemotherapy for metastatic colorectal cancer (mCRC) is mainly based on 5-FU with folinic acid (FA), preferably given as 24-48 h infusion, or oral prodrugs (e.g. capecitabine) in combination with either oxaliplatin or irinotecan [12]. Several studies with different chemo doublets could show median overall and progression-free survival ranging from 15 to 23 and 7 to 14 months, respectively, in patients with metastatic colorectal cancer (Table 1). Recently, Falcone et al. has shown a triple chemotherapy regimen combining 5-FU/FA, oxaliplatin and irinotecan (FOLFOXIRI) to be superior to FOLFIRI as first-line therapy [13]. In addition, triplets including targeted therapy such as antibodies against the vascular endothelial growth factor, VEGF (bevacizumab) or the epidermal growth factor receptor EGFR (cetuximab or panitumab) have been proven to be efficient in terms of prolonged overall and diseasefree survival in first line mCRC treatment [14]. Thus, PFS reached up to 12 months and OS ranged from 17 to 30 months (Tables 1 and 2). Nevertheless, the efficacy of the different triplet regimens may depend on tumor biology-related factors (e.g. histology, dissemination pattern, KRAS or BRAF mutation, anticipated chemosensitivity and growth dynamics). However, triplets are recommended by the recently published ESMO Consensus Guidelines for first-line treatment or induction therapy for most of patients with advanced colorectal cancer [12].

\section{EGFR-targeted therapy for advanced colorectal cancer}

The addition of targeted anticancer drugs against the epidermal growth factor receptor (EGFR), the monoclonal antibodies cetuximab and panitumumab, has further improved patient outcome in advanced stage colorectal cancer (Tables 2). Two prospective trials showed a survival benefit by adding cetuximab to best supportive care in patients with chemotherapy-refractory mCRC leading to a median OS of 6.4 and 6.1 months, respectively [38,39]. The BOND trial assigned patients with disease progression within three months after irinotecan-based chemotherapy to receive cetuximab with or without irinotecan. The median OS was 8.6 and 6.9 months, the time to progression 4.1 and 1.5 months, respectively [40]. In the randomized phase III CRYSTAL study investigating first-line treatment of mCRC the median PFS in the wild-type KRAS subgroup was 9.9 months in the FOLFIRI/cetuximab arm versus 8.7 months in the FOLFIRI arm. Median OS was 24.9 and 21 months, respectively. In patients with mutant KRAS status $(\mathrm{n}=192)$ median PFS was reduced after additional treatment with cetuximab (7.6 vs. 8.1 months) [33,41]. Similar observations are reported by Bokemeyer et al. after subgroup analysis of the prospective randomized OPUS study. The median progression-free survival rate was 7.2 months in both treatment arms with a 0.5 months benefit for additional treatment with cetuximab in the wild-type KRAS subgroup [34]. The results have been confirmed by a recently published pooled analysis of the CRYSTAL and OPUS trials [42].

Moreover, these observations are supported by the PRIME study that showed a significant improvement of PFS of untreated patients with wild-type KRAS mCRC by adding the EGFR antibody panitumumab to FOLFOX-4. Median PFS was 9.6 months in the panitumumab group vs. 8.0 months in the control group. There was also a nonsignificant benefit in overall survival (23.9 vs. 19.7 months) [28]. Another prospective randomized phase III study showed an increased PFS after adding panitumumab to FOLFIRI in second-line treatment of patients with mCRC (5.9 vs. 3.9 months) [43].

In contrast, the MRC COIN trial investigating the addition of cetuximab to an oxaliplatin-based chemotherapy for first-line treatment of patients with advanced CRC could not reproduce these findings. Although the response rate increased from $57 \%$ to $64 \%$ by adding cetuximab there was no significant benefit in median OS (17.9 in the control group vs. 17.0 months in the cetuximab group) as well as PFS (8.6 vs. 8.6 months). Nevertheless, in the subgroup analysis the lack of benefit was only reported for oxaliplatin and fluoropyrimidine combinations plus cetuximab in 
Table 1 Selected RCTs for systemic chemotherapy of advanced colorectal cancer

\begin{tabular}{|c|c|c|c|c|}
\hline Author, year & Pat. [n] & Treatment regimen & Median PFS [months] & Median OS [months] \\
\hline DeGramont, 2000 [15] & 210 & FOLFOX4 & 9.0 & 16.2 \\
\hline Giacchetti, 2000 [16] & 100 & FOLFOX & 8.7 & 19.4 \\
\hline Douillard, 2000 [17] & 199 & FOLFIRI & 6.7 & 17.4 \\
\hline Saltz, 2000 [18] & 226 & $\mathrm{IFL}$ & 7.0 & 14.8 \\
\hline Tournigand, 2003 [19] & 109 & FOLFIRI/FOLFOX6 & 14.2 & 21.5 \\
\hline Hurwitz, 2004 [20] & 402 & IFL/bevacizumab & 10.6 & 20.3 \\
\hline Goldberg, 2006 [21] & 154 & FOLFOX4 & 9.7 & 19.0 \\
\hline Falcone, 2007 [13] & 122 & FOLFOXIRI & 9.8 & 22.6 \\
\hline Fuchs, $2008[22,23]$ & 57 & FOLFIRI/bevacizumab & 11.2 & 28.0 \\
\hline Saltz, 2008 [24] & 699 & FOLFOX4/bevacizumab & 9.4 & 21.3 \\
\hline \multirow[t]{2}{*}{ Hecht, 2009 [25] } & 823 & FOLFOX/FOLFIRI/bev & 11.4 & 24.5 \\
\hline & & + panitumumab & 10.0 & 19.4 \\
\hline Tebbutt, 2010 [26] & 157 & capecitabine/bevacizumab & 8.5 & - \\
\hline \multirow[t]{2}{*}{ Stathopoulos, 2010 [27] } & 114 & IRI/5-FU/FA/bevacizumab & - & 22.0 \\
\hline & 108 & IRI/5-FU/FA & - & 25.0 \\
\hline Douillard, 2010 [28] & 1183 & FOLFOX4/panitumumab & 9.6 & 23.9 \\
\hline
\end{tabular}

contrast to combinations with infusional 5-FU [36]. In the recently published NORDIC-VII trial no benefit could be shown for the addition of cetuximab to an oxaliplatinbased combination with bolus 5-FU only (FLOX). In the ITT analysis the median progression-free survival was 7.9 months in the control group vs. 8.3 months in the cetuximab group, respectively [37].

\section{Systemic treatment of colorectal PM}

Due to the fact that peritoneal metastases (PM) differ from other metastatic sites regarding clinical course and prognosis, Franko et al. published a pooled subgroup analysis of 364 patients with mCRC from the two North Central Cancer Treatment Group phase III trials N9741 and N9841. The patients were treated with oxaliplatinor irinotecan-based systemic chemotherapy. The 5-year OS was $4.1 \%$ in patients with (additional) peritoneal metastases (PM) vs. $6 \%$ in patients without PM, showing a $30 \%$ relative reduction of OS in case of peritoneal carcinomatosis. Systemic chemotherapy with FOLFOX was superior to irinotecan-based treatment regimens, irrespective of the carcinomatosis status [44]. Klaver et al. analyzed the survival of the subgroup of patients with PM at the time of enrolment in the prospective clinical trials CAIRO and CAIRO2. The median OS significantly decreased in the PM group compared to patients without peritoneal tumor spread (CAIRO: 10.4 vs. 17.3 months, CAIRO2: 15.2 vs. 20.7 months) [45]. In 63 patients with colorectal PM selected from the French database that received several regimens of modern systemic chemotherapy the median OS was 23.9 months [46]. An Asian prospective single-arm phase II study investigating FOLFOX-4 in patients with peritoneal metastases from CRC reported a median time to progression of 4.4 months and a median overall survival of 21.5 months [47].

Table 2 Cetuximab for advanced colorectal cancer

\begin{tabular}{|c|c|c|c|c|c|c|}
\hline \multirow[t]{2}{*}{ Author, year } & \multirow{2}{*}{$\begin{array}{l}\text { Pat. } \\
\text { [n] }\end{array}$} & \multirow{2}{*}{$\begin{array}{l}\text { Treatment } \\
\text { regimen }\end{array}$} & \multicolumn{2}{|c|}{ Median PFS [months] } & \multicolumn{2}{|c|}{ Median OS [months] } \\
\hline & & & + cet & - cet & + cet & - cet \\
\hline Tabernero, 2007 [29] & 43 & FOLFOX/cetuximab & 12.3 & - & 30.5 & - \\
\hline Borner, 2008 [30] & 74 & CAPOX/cetuximab & 7.2 & 5.8 & 20.5 & 16.5 \\
\hline Arnold, 2008 [31] & 49 & FUFOX/cetuximab & 8.1 & - & 28.2 & - \\
\hline Cartwright, 2008 [32] & 70 & CAPIRI/cetuximab & 8.1 & - & 20.5 & - \\
\hline Van Cutsem, 2009 [33] & $348^{*}$ & FOLFIRI/cetuximab & $9.9^{*}$ & $8.7^{*}$ & $24.9^{*}$ & $21^{*}$ \\
\hline Bokemeyer, 2009 [34] & $134^{*}$ & FOLFOX/cetuximab & $7.7^{*}$ & $7.2^{*}$ & - & - \\
\hline Tol, 2009 [35] & 755 & CAPOX/bev/cet & 9.4 & 10.7 & 19.4 & 20.3 \\
\hline Maughan, 2011 [36] & $729^{*}$ & FOLFOX/CAPOX/cet & $8.6^{*}$ & $8.6^{*}$ & $17.0^{*}$ & $17.9^{*}$ \\
\hline Tveit, 2012 [37] & 566 & FLOX/cetuximab & 8.3 & 7.9 & 19.7 & 20.4 \\
\hline
\end{tabular}

\footnotetext{
*wild-type KRAS
} 


\section{Cytoreductive surgery and HIPEC}

The combined treatment concept of cytoreductive surgery (CRS) and hyperthermic intraperitoneal chemotherapy (HIPEC) was introduced by Sugarbaker et al. in the early 1990's and consists of complete macroscopic cytoreduction of all visible tumor nodules followed by local intraabdominal chemoperfusion at $41-42^{\circ} \mathrm{C}[48,49]$. The aim of HIPEC in patients with peritoneal carcinomatosis is to circumvent the peritoneal barrier and to obtain higher local concentration of the cytostatic agents [50-52]. However, until today the intraperitoneal or bidirectional chemotherapeutic regimen is not standardized [53-56]. The addition of hyperthermia may potentiate the effect of the cytostatic agents by thermic cytotoxicity and induction of apoptosis. Moreover, heating can improve tissue penetration of the cytostatic agents $[48,57,58]$.

Numerous retrospective analyses reported feasibility, safety and efficacy of the combined treatment concept of CRS and HIPEC in patients with peritoneal carcinomatosis arising from CRC (Table 3). However, data from prospective trials are still limited. Verwaal et al. reported a prospective randomized phase III trial analyzing CRS and HIPEC with MMC plus adjuvant chemotherapy with 5-FU/folinic acid compared to systemic chemotherapy with 5-FU/folinic acid and palliative surgery, if possible. After a median follow-up of 21.6 months, the experimental treatment arm showed a median overall survival of 22.3 months compared to 12.6 months in the standard arm. In the subgroup of patients with complete macroscopic cytoreduction (CC-0/1) median survival was 42.9 months. Median progression-free survival was 12.6 and 7.7 months, respectively $[59,60]$. Another randomized controlled trial was launched by a French group. This study published by Elias et al. was designed to compare CRS with early postoperative intraperitoneal chemotherapy (EPIC) to CRS alone. After premature termination due to recruitment difficulties a 2-year survival rate of $60 \%$ was reported in 35 patients with complete macroscopic cytoreduction [61]. In the comparative study published by Mahteme et al. the median survival in the HIPEC group was 32 months vs. 14 months in the control group. 5-year survival rates were $28 \%$ and $5 \%$ respectively [62]. A multi- center registry study of 506 patients treated with CRS and HIPEC for peritoneal carcinomatosis arising from colorectal cancer reported median overall survival of 19.2 months. In patients with complete macroscopic cytoreduction (CC$0 / 1$ ) the median survival was 32.4 months [63]. In numerous observational studies the overall median survival ranged from 15 to 32 months and from 28 to 60 months after complete macroscopic cytoreduction (CC-0/1), respectively [64]. Elias et al. compared 48 patients from the French Multicenter Database with peritoneal carcinomatosis arising from CRC who received palliative systemic chemotherapy to 48 patients who underwent additional CRS and bidirectional oxaliplatin-based HIPEC. The chemotherapeutic regimen and the duration of systemic chemotherapy were comparable in both groups. The median survival was 23.9 months in the control group vs. 62.7 months in the HIPEC group, and the 5-year survival rate was $13 \%$ and $51 \%$, respectively [46]. Comparable results were obtained in a recently published Belgian prospective multicenter phase II study in 48 consecutive patients with CRC and peritoneal carcinomatosis after CRS and oxaliplatin-based HIPEC. Hompes et al. reported a median time until recurrence of 19.8 months, and a 2year overall survival rate of $88.7 \%$ [65]. The differences in median survival of the control group between these analyses and the Dutch Trial may be explained by patient selection and the introduction of more efficient combined chemotherapeutic regimens with or without targeted drugs in the standard treatment of advanced stage CRC.

\section{Methods/design \\ Study design}

The COMBATAC study is a prospective, multicenter, open-label, single-arm, single-stage phase II study. The investigator initiated trial (IIT) is conducted by the Department of Surgery of the University Medical Center Regensburg in collaboration with the Center for Clinical Studies Regensburg, the Coordination Centre for Clinical Trials Duesseldorf and the participating national peritoneal carcinomatosis centers.

The study protocol is supported by the CRC Study Group of the Arbeitsgemeinschaft Internistische Onkologie

Table 3 CRS and HIPEC

\begin{tabular}{lcccccc}
\hline Author, year & Pat. [n] & Cytostatic agents (HIPEC) & Median OS [mths] & Median PFS [mths] & OS [\%] & Survival CC-0/1 [\%] \\
\hline Pilati, 2003 [66] & 34 & MMC/DDP & 18 & - & $31(2-y)$ & - \\
\hline Glehen, 2004 [67] & 53 & MMC & 13 & - & $32(2-y)$ & $54(2-y)$ \\
\hline Glehen, 2004 [63] & 506 & MMC/LOHP & 19 & - & $39(3-y)$ & $47(3-y)$ \\
\hline Shen, 2004 [68] & 77 & MMC & 16 & - & $25(3-y)$ & $44(3-y)$ \\
\hline Verwaal, 03/08 [59,60] & 105 & MMC & 22 & 12.6 & $28(3-y)$ & $45(5-y)$ \\
\hline Quenet, ASCO 08 [69] & 37 & LOHP/IRI & 37 & - & - & $58(3-y)$ \\
\hline Elias, 2009 [46] & 48 & LOHP & 63 & 13 & $51(5-y)$ \\
\hline Hompes, 2012 [65] & 48 & LOHP & - & - & $89(2 y)$ \\
\hline
\end{tabular}


(AIO) and the Chirurgische Arbeitsgemeinschaft Onkologie $(\mathrm{CAO}-\mathrm{V})$ of the German Society of General and Visceral Surgery (DGAV).

\section{Study objectives and endpoints}

The primary objective of the COMBATAC study in patients with peritoneal carcinomatosis arising from wildtype KRAS colorectal and appendiceal cancer is to estimate the progression-free survival (PFS). Based on this estimation, it will be determined whether the multimodality treatment with pre- and postoperative systemic chemotherapy plus cetuximab, cytoreductive surgery (CRS) and bidirectional hyperthermic intraoperative chemotherapy (HIPEC) shows sufficient evidence of efficacy for further investigation.

PFS is defined as the time interval between the first day of preoperative treatment and the date of progression or death, whichever occurs first. Patients who are alive and progression-free at the time of analysis will be censored for PFS at the time of their last contact.

Secondary endpoints include overall survival, morbidity and toxicity related to the locoregional approach, feasibility of the combined treatment concept, quality of life and pathohistological regression.

\section{Study population}

The study population of the COMBATAC study consists of patients with synchronous or metachronous peritoneal carcinomatosis arising from histologically proven wildtype KRAS colorectal or appendiceal cancer. The extent of peritoneal tumor spread (Peritoneal cancer Index, PCI) as assessed by diagnostics such as computed tomography and laparoscopy prior to patient enrolment should allow complete macroscopic cytoreduction (CC-0/1) at the time of surgery. Moreover, patients to be included in the study must meet the following inclusion criteria: treatment-free interval of at least 6 months after the completion of 3prior systemic chemotherapy, age over 18 and below 71 years, good general health status (Karnofsky index more than $70 \%$, ECOG 0-2), absence of hematogenous metastases (lung, bone, brain, $>3$ peripheral resectable liver metastases), absence of contraindication for systemic chemotherapy and/or extended surgery, estimated life expectancy more than 6 months, absence of any psychological, familial, sociological or geographical condition potentially hampering compliance with the study protocol and follow-up schedule, written informed consent, creatinine clearance $>50 \mathrm{ml} / \mathrm{min}$, serum creatinine $\leq 1.5 \times \mathrm{ULN}$, serum bilirubin $\leq 1.5 \times \mathrm{ULN}$, ASAT and ALAT $\leq 2.5 \times \mathrm{ULN}$, platelet count $>100,000 / \mathrm{ml}$, haemoglobin $>9 \mathrm{~g} / \mathrm{dl}$, neutrophil granulocytes $\geq 1,500 / \mathrm{ml}$, International Normalized Ration $(\mathrm{INR}) \leq 2$, absence of peripheral neuropathy $>$ grade 1 (CTCAE version 4.0), no pregnancy or breast feeding and adequate contraception in fertile patients. Patients with incomplete cytoreduction ( $\geq \mathrm{CC}-2$ ), tumor debulking or palliative surgery, hematogenous metastasis excluding less than three resectable liver metastases and/or prior chemotherapy $<6$ months before evaluation of study inclusion or therapy with EGFR receptor antibody for metastatic disease are excluded from the present study. Further exclusion criteria are KRAS mutation, known allergy to murine or chimeric monoclonal antibodies, concurrent chronic systemic immune therapy, chemotherapy, or hormone therapy not indicated in the study protocol, histology of signet ring carcinoma ( $>20 \%$ of tumor cells), other malignancy than disease under study or second cancer $<5$ years after R0 resection, impaired liver, renal or hematologic function as mentioned above, heart failure NYHA $\geq 2$ or significant coronary artery disease (CAD), alcohol and/or drug abuse, inclusion in other clinical trials interfering with the study protocol. Patients can only be included once in the COMBATAC study.

\section{Treatment schedule}

The interdisciplinary combined treatment regimen consists of pre- and postoperative systemic chemotherapy with FOLFOX or FOLFIRI plus the EGFR antagonist cetuximab, cytoreductive surgery (CRS) with complete macroscopic cytoreduction (CC-0/1) followed by bidirectional hyperthermic intraperitoneal chemotherapy (HIPEC). The treatment schedule is shown in Figure 1.

Systemic chemotherapy will consist of standard-of-care chemotherapy. Preoperative intravenous chemotherapy will be applied for 3 months, and therapy will be completed by postoperative systemic chemotherapy for further 3 months starting 4-6 weeks after surgery. Cetuximab is given intravenously once weekly for max. 12 weeks. The initial dose is $400 \mathrm{mg} / \mathrm{m}^{2}$ body surface area followed by a weekly dose of $250 \mathrm{mg} / \mathrm{m}^{2}$. Standard of care premedication will be administered as needed to patients receiving intravenous chemotherapy, including dexamethasone, acid suppressors, anti-emetics, analgetics and antipyretics. Systemic chemotherapy will be administered by the patients' medical oncologist or the department of oncology of the enrolling peritoneal carcinomatosis center. All decisions regarding the management of (serious) adverse events related to systemic chemotherapy, such as dose reduction, interruption of systemic treatment or change of treatment regimen are at the discretion of the treating medical oncologist and are allowed within the study protocol, if documented.

Preoperative systemic chemotherapy is followed by cytoreductive surgery and HIPEC. The intent of cytoreductive surgery is to obtain complete macroscopic cytoreduction (CC-0/1) as a precondition for the application of HIPEC. The residual disease is classified intraoperatively using the completeness of cytoreduction (CC) score. $\mathrm{CC}-0$ indicates no visible residual tumor and $\mathrm{CC}-1$ 


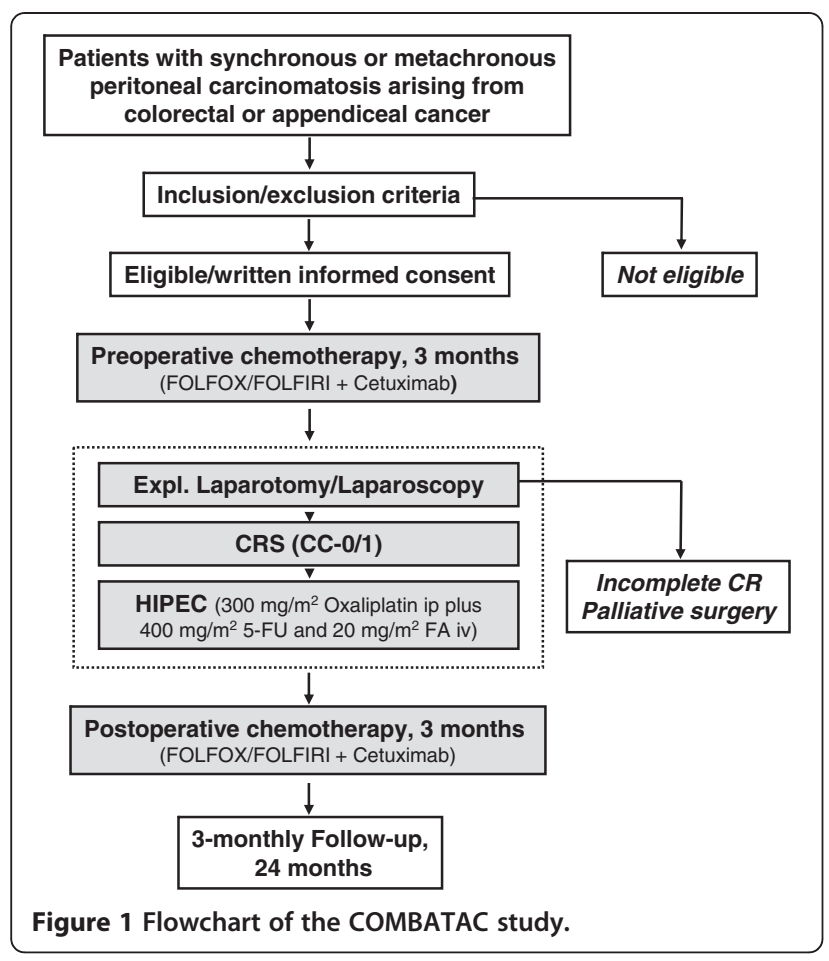

residual tumor nodules $\leq 2.5 \mathrm{~mm}$. CC-2 and CC-3 indicate residual tumor nodules between $2.5 \mathrm{~mm}$ and $2.5 \mathrm{~cm}$ and $>2.5 \mathrm{~cm}$, respectively [70]. The initial extent of peritoneal tumor manifestation is determined intraoperatively using the Peritoneal Cancer Index (PCI, Washington Cancer Center), a combined numerical score of lesion size (LS-0 to LS-3) and tumor localization (region 0-12) $[70,71]$. During surgery patients are placed in modified lithotomy position. Surgery may include parietal and visceral peritonectomy, greater omentectomy, splenectomy, cholecystectomy, resection of liver capsule, small bowel resection, colonic and rectal resection, (subtotal) gastrectomy, lesser omentectomy, pancreatic resection, hysterectomy, ovariectomy and urine bladder resection. In patients with infiltration of the umbilicus, omphalectomy is necessary. Further operating procedures and resections may be necessary due to the intraoperative findings. Gastrointestinal reconstructions are performed following the individual center's standard operating procedures (SOPs). The following minimal requirements are prerequisites for CRS: complete greater omentectomy, complete adhesiolysis of the small intestine, complete mobilization of the liver to assess the right diaphragmatic space, assessment of the left diaphragmatic space requiring splenectomy in the majority of cases, assessment of the left and right paracolic spaces, assessment of the pelvis, often requiring anterior rectal resection.

Bidirectional oxaliplatin-based hyperthermic intraperitoneal chemoperfusion (HIPEC) will only be applied intraoperatively in case of complete macroscopic cytoreduction
(CC-0/1). HIPEC may be performed in an open or closed abdomen technique according to the peritoneal carcinomatosis center's SOPs. After CRS four intraabdominal drains and two temperature probes are placed for continuous abdominal perfusion using a roller pump system with heat exchanger as described before [72]. When Douglas pouch temperature reaches $40^{\circ} \mathrm{C}$ oxaliplatin at a concentration of $300 \mathrm{mg} / \mathrm{m}^{2}$ body surface area is added and perfusion will be continued for further 30 minutes. The treatment is combined with synchronous IV administration of $400 \mathrm{mg} / \mathrm{m}^{2}$ fluorouracil and $20 \mathrm{mg} / \mathrm{m}^{2}$ folinic acid considering toxicity and safety instructions. After completion of the intraperitoneal perfusion cycle, the perfusion volume is evacuated from the abdominal cavity, all drains remain in situ and the patient is transferred to postoperative care.

\section{Assessments and follow-up}

During the screening period patients will be assessed for eligibility to be included in the COMBATAC study. Inclusion and exclusion criteria are assessed by the investigator and initial diagnostics will be completed as necessary prior to patient enrolment. During pre- and postoperative systemic chemotherapy clinical examination and laboratory testing will be performed within 7 days of each chemotherapy cycle. After completion of preoperative treatment and after completion of the postoperative chemotherapy (end of treatment period), a further staging computed tomography will be performed. Moreover, quality of life is assessed and tumor markers (CEA, CA19-9) are determined. The same items will be recorded within three weeks after surgery. Intraoperative data consisting of PCI, surgical procedures, number of anastomoses, operating time, blood loss and course of HIPEC procedure and additional postoperative such as stay on ICU and hospital stay will be documented. The follow-up time starts 30 days after the last day of drug administration during postoperative treatment with the 'end-of-treatment' visit. The follow-up time takes 24 months with three-monthly follow-up visits consisting of physical examination, laboratory testing including tumor markers and protocol CT scans. Quality of life will be assessed yearly during follow-up.

Radiological disease progression will be assessed according to the revised RECIST criteria version 1.1 [73]. As mentioned above, computed tomography of the chest, abdomen and pelvis with oral, rectal and intravenous contrast will be performed prior to treatment start, within 3 weeks after cytoreductive surgery (CRS) and HIPEC and 30 days after the last systemic drug administration. Response to treatment is defined by the following four categories. (1) complete response (CR), (2) partial response (PR, 30\% decrease in sum of baseline), (3) stable disease (SD) and (4) progressive disease (PD, new lesions or $20 \%$ increase in sum from nadir). Determination of disease 
progression in the absence of lymphatic or hematogenous disease recurrence will be based on clinical signs or symptoms (e.g. malignant ascites, ureteral stenosis or bowel obstruction), radiological diagnosis (CT $\pm \mathrm{PET}$ ) and/or surgical evidence of progression during laparoscopy or laparotomy. In addition, CEA and CA19-9 will be routinely measured as mentioned above. An at least three fold increase in serum CEA or CA19-9 levels will be defined as progression.

Morbidity and toxicity will be assessed as the number of medical and surgical complications occurring during the treatment period. The severity of complications (Grade I-V) will be assessed and adverse events will be categorized using the CTCAE version 4.0 [74].

Quality of life will be assessed using the EORTC QLQC30 questionnaire. Functional and symptom scores will be calculated according to the standard scoring procedures [75]. Comparisons will be drawn with the score means of the reference population [76]. A second round of analyses will be performed in order to identify the proportion of patients at any assessment point with pronounced deficits in QoL as defined by score points $<50$ on a $0=$ very bad to $100=$ very good scale [77].

The pathohistological regression after systemic chemotherapy is assessed and graded using the classification published by Dworak et al [78]. This classification system was originally generated to evaluate regression of rectal cancer after neoadjuvant radiotherapy and consists of different types of necrosis and fibrosis with specific changes of vascular and cellular morphology.

\section{Statistical considerations}

The sample size was calculated using the primary endpoint, i.e. progression-free survival (PFS). Based on the literature, a median PFS of 10 months or less was considered to be of no further interest (treatment not promising). Alpha (one-sided) was set to $10 \%$ and Beta was set to $20 \%$ (acceptable error rates for phase II trials [79]). Assuming exponentially distributed progression times and a target median PFS of 14 months (treatment promising), at least 39 events (progressions or deaths) have to be observed. Equivalently, if the true median PFS is 14 months, 39 events will be sufficient to rule out a median PFS of 10 months, based on the one-sided $90 \%$ confidence interval $(\mathrm{CI})$. The normal approximation used in the calculations is given by equation 3.2.7 of Lawless [80]. Assuming an accrual period of 12 months and a follow-up of at least 18 months from the last patient recruited, a minimum number of 51 patients will be required. With a lost-tofollow-up rate of maximal 15\%, a total of 60 patients have to be included in the study.

The final analysis with respect to PFS will be done after 39 observed events. PFS distribution and median PFS time with the corresponding one-sided $90 \%$ CI will be estimated by means of the Kaplan-Meier method. The treatment will be considered worth further investigation if the lower bound of the CI is greater than 10 months. The primary analysis will be based on the intention-to-treat (ITT) analysis set that consists of all patients who entered the study.

A detailed description of statistical analysis methods will be given in the Statistical Analysis Plan which will be finalized prior to database lock.

\section{Data collection and quality assurance}

Patient data are collected in an electronic case report form (eCRF) at the data centre of the Center for Clinical Studies Regensburg in collaboration with the Coordination Centre for Clinical Trials Duesseldorf. Consistency checks will be performed on newly entered forms and queries issued in case of inconsistencies. Archiving of trial documents and trial data is performed according to the internal SOPs of the Center for Clinical Studies Regensburg. The originals of all essential trial documents are filed in the Trial Master File (TMF) and archived for at least 15 years. The sitespecific documents in the Investigator Site File (ISF) will be archived at the site for at least 15 years. On-site monitoring will be performed by an external CRO (multiservice-monitoring, Regensburg, Germany) adapted according to the site accrual.

\section{Ethical and legal aspects}

The protocol will be conducted according to the guidelines of Good Clinical Practice (GCP) and the ethical principles described in the Declaration of Helsinki. The study protocol was approved by the leading ethic committee (Ethikkommission an der Universitaet Regensburg) and the associated ethics committees, and was also subject to authorization by the national competent authority (BfArM) as mandatory by federal law. The study was assigned the EudraCT number 2009-014040-11 and is registered at ClinicalTrials.gov (NCT01540344).

\section{Discussion}

The COMBATAC study is designed to evaluate the feasibility and efficacy of CRS and bidirectional oxaliplatinbased HIPEC as an additional treatment option for selected patients within an interdisciplinary combined treatment concept consisting of standard-of care pre- and postoperative systemic chemotherapy.

It is beyond question that systemic chemotherapy is the standard of care in patients with advanced stage CRC and peritoneal carcinomatosis. Although the oncological outcome of patients with advanced stage CRC and also the subgroup of patients with peritoneal carcinomatosis has improved since the introduction of combined chemotherapeutic regimens and new drugs, results of systemic therapy for patients with peritoneal carcinomatosis are still unsatisfactory [44]. Thus, additional treatment options 
should be evaluated. The existing data show that CRS and HIPEC may improve long-term survival of selected patients with peritoneal carcinomatosis of colonic origin $[59,60]$. Moreover, hyperthermic peritoneal perfusion with oxaliplatin in combination with synchronous intravenous application of 5-FU/folinic acid seems to improve the efficacy of HIPEC in comparison to a mitomycin C-based intraperitoneal treatment regimen, and may additionally contribute to a better local disease control $[46,65]$. Perioperative morbidity and mortality seems not to be impaired by the intensified oxaliplatin-based HIPEC regimen [81]. Nevertheless, the time of surgery including HIPEC, the perioperative treatment and the sequence of the therapeutic interventions is still a matter of debate. The intensified systemic treatment strategy with preoperative chemotherapy may lead to increased rates of complete macrosopic cytoreduction and together with the postoperative treatment to better control of distant metastasis and tumor recurrence. However, there is no prospective study available evaluating the clinical and oncological outcome after standard-of-care chemotherapy including targeted anticancer therapy in combination with CRS and HIPEC. Thus, the COMBATAC study is expected to give further information about the efficacy of this promising therapeutic option as an inherent part of a multidisciplinary treatment concept.

\section{Conclusions}

To our knowledge the COMBATAC study is the first prospective clinical trial investigating the feasibility and efficacy of CRS and bidirectional oxaliplatin-based HIPEC within an interdisciplinary treatment regimen with pre- and postoperative systemic chemotherapy including cetuximab.

\section{Abbreviations}

5-FU: Fluorouracil; BfArM: Bundesinstitut fuer Arzneimittel und Medizinprodukte; CA19-9: Carbohydrate antigen 19-9; CAPIRI: Capecitabine + irinotecan; CAPOX: Capecitabine + oxaliplatin; CEA: Carcinoembryonic antigen; COMBATAC: COMBined Anticancer Treatment of Advanced Colorectal cancer; CRC: Colorectal cancer; CRO: Contract research organization; CRS: Cytoreductive surgery; CT: Computed tomography; CTCAE: Common Terminology Criteria for Adverse Events; DDP: Cisplatin; EGFR: Epithelial growth factor receptor; EORTC: European Organisation for Research and Treatment of Cancer; EudraCT: European Clinical Trial Database; FA: Folinic acid; FOLFIRI: Folinic acid + fluorouracil + irinotecan; FOLFOX: Folinic acid + fluorouracil + oxaliplatin; HIPEC: Hyperthermic intraperitoneal chemotherapy; IFL: Irinotecan + fluorouracil + leucovorin; IIT: Investigator initiated trial; IRI: Irinotecan; ITT: Intention-to-treat; KRAS: Kirsten rat sarcoma viral oncogene homolog; LOHP: Oxaliplatin; mCRC: Metastatic colorectal cancer; MMC: Mitomycin C; MRI: Magnetic resonance imaging; OS: Overall survival; PCl: Peritoneal Cancer Index; PET: Positron emission tomography; PFS: Progression-free survival; PM: Peritoneal metastases; PP: Per protocol; QoL: Quality of life; RCT: Randomized controlled trial; SOPs: Standard operating procedures; ULN: Upper limit of normal.

\section{Competing interests}

The COMBATAC study is financially supported by Merck KGaA, Darmstadt, Germany. GG, JR, SL, FZ and MK have nothing to declare. DA, FK, HJS and PP received honoraria from Merck KGaA.

\section{Authors' contributions}

GG drafted the manuscript and the study protocol. JR, DA, SAL, FK, FZ, MK, HJS and PP participated in writing the study protocol and revised the manuscript. PP is the principal and coordinating investigator of the COMBATAC trial. All authors read and approved the final manuscript.

\section{Author details}

'Department of Surgery, University Medical Center Regensburg, Regensburg 93042, Germany. ${ }^{2}$ Institute of Medical Biometry and Informatics, University of Heidelberg, Heidelberg, Germany. ${ }^{3}$ Department of Medical Oncology, Tumor Biology Clinic, Albert Ludwigs University, Freiburg, Germany. ${ }^{4}$ Department of Internal Medicine, University Medical Center Regensburg, Regensburg, Germany. ${ }^{5}$ Center for Clinical Studies, University Medical Center Regensburg, Regensburg, Germany. ${ }^{6}$ Department of Surgery, St. John of God Hospital Regensburg, Regensburg, Germany.

Received: 12 November 2012 Accepted: 4 February 2013 Published: 7 February 2013

\section{References}

1. Jemal A, Bray F, Center MM, Ferlay J, Ward E, Forman D: Global cancer statistics. CA Cancer J Clin 2011, 61(2):69-90.

2. Ferlay J, Shin HR, Bray F, Forman D, Mathers C, Parkin DM: Estimates of worldwide burden of cancer in 2008: GLOBOCAN 2008. Int J Cancer 2010, 127(12):2893-2917.

3. Glehen O, Osinsky D, Cotte E, Kwiatkowski F, Freyer G, Isaac S, Trillet-Lenoir V, Sayag-Beaujard AC, Francois Y, Vignal J, et al: Intraperitoneal chemohyperthermia using a closed abdominal procedure and cytoreductive surgery for the treatment of peritoneal carcinomatosis: morbidity and mortality analysis of 216 consecutive procedures. Ann Surg Oncol 2003, 10(8):863-869.

4. Russell AH, Tong D, Dawson LE, Wisbeck W: Adenocarcinoma of the proximal colon. Sites of initial dissemination and patterns of recurrence following surgery alone. Cancer 1984, 53(2):360-367.

5. Willett CG, Tepper JE, Cohen AM, Orlow E, Welch CE: Failure patterns following curative resection of colonic carcinoma. Ann Surg 1984, 200(6):685-690.

6. Schott A, Vogel I, Krueger U, Kalthoff H, Schreiber HW, Schmiegel W, HenneBruns D, Kremer B, Juhl H: Isolated tumor cells are frequently detectable in the peritoneal cavity of gastric and colorectal cancer patients and serve as a new prognostic marker. Ann Surg 1998, 227(3):372-379.

7. Carmignani CP, Sugarbaker TA, Bromley CM, Sugarbaker PH: Intraperitoneal cancer dissemination: mechanisms of the patterns of spread. Cancer Metastasis Rev 2003, 22(4):465-472.

8. Ceelen WP, Bracke ME: Peritoneal minimal residual disease in colorectal cancer: mechanisms, prevention, and treatment. Lancet Oncol 2009, 10(1):72-79.

9. Stewart JH, Shen P, Levine EA: Intraperitoneal hyperthermic chemotherapy for peritoneal surface malignancy: current status and future directions. Ann Surg Oncol 2005, 12(10):765-777.

10. Sadeghi B, Arvieux C, Glehen O, Beaujard AC, Rivoire M, Baulieux J, Fontaumard E, Brachet A, Caillot JL, Faure JL, et al: Peritoneal carcinomatosis from non-gynecologic malignancies: results of the EVOCAPE 1 multicentric prospective study. Cancer 2000, 88(2):358-363.

11. Jayne DG, Fook S, Loi C, Seow-Choen F: Peritoneal carcinomatosis from colorectal cancer. Br J Surg 2002, 89(12):1545-1550.

12. Schmoll HJ, Van Cutsem E, Stein A, Valentini V, Glimelius B, Haustermans K Nordlinger B, van de Velde CJ, Balmana J, Regula J, et al: ESMO consensus guidelines for management of patients with colon and rectal cancer. A personalized approach to clinical decision making. Ann Oncol 2012, 23(10):2479-2516.

13. Falcone A, Ricci S, Brunetti I, Pfanner E, Allegrini G, Barbara C, Crino L, Benedetti $G$, Evangelista W, Fanchini $L$, et al: Phase III trial of infusional fluorouracil, leucovorin, oxaliplatin, and irinotecan (FOLFOXIRI) compared with infusional fluorouracil, leucovorin, and irinotecan (FOLFIRI) as first-line treatment for metastatic colorectal cancer: the Gruppo Oncologico Nord Ovest. J Clin Oncol 2007, 25(13):1670-1676.

14. Arnold D, Seufferlein $T$ : Targeted treatments in colorectal cancer: state of the art and future perspectives. Gut 2010, 59(6):838-858.

15. de Gramont A, Figer A, Seymour M, Homerin M, Hmissi A, Cassidy J, Boni C, Cortes-Funes H, Cervantes A, Freyer $\mathrm{G}$, et al: Leucovorin and fluorouracil 
with or without oxaliplatin as first-line treatment in advanced colorectal cancer. J Clin Oncol 2000, 18(16):2938-2947.

16. Giacchetti S, Perpoint B, Zidani R, Le Bail N, Faggiuolo R, Focan C, Chollet P, Llory JF, Letourneau Y, Coudert B, et al: Phase III multicenter randomized trial of oxaliplatin added to chronomodulated fluorouracil-leucovorin as first-line treatment of metastatic colorectal cancer. J Clin Oncol 2000 18(1):136-147.

17. Douillard JY, Cunningham D, Roth AD, Navarro M, James RD, Karasek P, Jandik P, Iveson T, Carmichael J, Alakl M, et al: Irinotecan combined with fluorouracil compared with fluorouracil alone as first-line treatment for metastatic colorectal cancer: a multicentre randomised trial. Lancet 2000, 355(9209):1041-1047.

18. Saltz LB, Cox JV, Blanke C, Rosen LS, Fehrenbacher L, Moore MJ, Maroun JA, Ackland SP, Locker PK, Pirotta N, et al: Irinotecan plus fluorouracil and leucovorin for metastatic colorectal cancer. Irinotecan Study Group. N Engl J Med 2000, 343(13):905-914.

19. Tournigand C, Andre T, Achille E, Lledo G, Flesh M, Mery-Mignard D, Quinaux E, Couteau C, Buyse M, Ganem G, et al: FOLFIRI followed by FOLFOX6 or the reverse sequence in advanced colorectal cancer: a randomized GERCOR study. J Clin Oncol 2004, 22(2):229-237.

20. Hurwitz H, Fehrenbacher L, Novotny W, Cartwright T, Hainsworth J, Heim W, Berlin J, Baron A, Griffing S, Holmgren E, et al: Bevacizumab plus irinotecan, fluorouracil, and leucovorin for metastatic colorectal cancer. N Engl J Med 2004, 350(23):2335-2342.

21. Goldberg RM, Sargent DJ, Morton RF, Fuchs CS, Ramanathan RK, Williamson SK, Findlay BP, Pitot HC, Alberts S: Randomized controlled trial of reduceddose bolus fluorouracil plus leucovorin and irinotecan or infused fluorouracil plus leucovorin and oxaliplatin in patients with previously untreated metastatic colorectal cancer: a North American Intergroup Trial. J Clin Oncol 2006, 24(21):3347-3353.

22. Fuchs CS, Marshall J, Barrueco J: Randomized, controlled trial of irinotecan plus infusional, bolus, or oral fluoropyrimidines in first-line treatment of metastatic colorectal cancer: updated results from the BICC-C study. J Clin Oncol 2008, 26(4):689-690.

23. Fuchs CS, Marshall J, Mitchell E, Wierzbicki R, Ganju V, Jeffery M, Schulz J, Richards D, Soufi-Mahjoubi R, Wang B, et al: Randomized, controlled trial of irinotecan plus infusional, bolus, or oral fluoropyrimidines in first-line treatment of metastatic colorectal cancer: results from the BICC-C Study. J Clin Oncol 2007, 25(30):4779-4786.

24. Saltz LB, Clarke S, Diaz-Rubio E, Scheithauer W, Figer A, Wong R, Koski S, Lichinitser M, Yang TS, Rivera F, et al: Bevacizumab in combination with oxaliplatin-based chemotherapy as first-line therapy in metastatic colorectal cancer: a randomized phase III study. J Clin Oncol 2008, 26(12):2013-2019.

25. Hecht JR, Mitchell E, Chidiac T, Scroggin C, Hagenstad C, Spigel D, Marshall J, Cohn A, McCollum D, Stella P, et al: A randomized phase IIIB trial of chemotherapy, bevacizumab, and panitumumab compared with chemotherapy and bevacizumab alone for metastatic colorectal cancer. J Clin Oncol 2009, 27(5):672-680.

26. Tebbutt NC, Wilson K, Gebski VJ, Cummins MM, Zannino D, van Hazel GA, Robinson B, Broad A, Ganju V, Ackland SP, et al: Capecitabine, bevacizumab, and mitomycin in first-line treatment of metastatic colorectal cancer: results of the Australasian Gastrointestinal Trials Group Randomized Phase III MAX Study. J Clin Oncol 2010, 28(19):3191-3198.

27. Stathopoulos GP, Batziou C, Trafalis D, Koutantos J, Batzios S, Stathopoulos J, Legakis J, Armakolas A: Treatment of colorectal cancer with and without bevacizumab: a phase III study. Oncology 2010, 78(5-6):376-381.

28. Douillard JY, Siena S, Cassidy J, Tabernero J, Burkes R, Barugel M, Humblet Y, Bodoky G, Cunningham D, Jassem J, et al: Randomized, phase III trial of panitumumab with infusional fluorouracil, leucovorin, and oxaliplatin (FOLFOX4) versus FOLFOX4 alone as first-line treatment in patients with previously untreated metastatic colorectal cancer: the PRIME study. J Clin Oncol 2010, 28(31):4697-4705.

29. Tabernero J, Van Cutsem E, Diaz-Rubio E, Cervantes A, Humblet $Y$, Andre T, Van Laethem JL, Soulie P, Casado E, Verslype C, et al: Phase II trial of cetuximab in combination with fluorouracil, leucovorin, and oxaliplatin in the first-line treatment of metastatic colorectal cancer. J Clin Oncol 2007, 25(33):5225-5232.

30. Borner M, Koeberle D, Von Moos R, Saletti P, Rauch D, Hess V, Trojan A Helbling D, Pestalozzi B, Caspar C, et al: Adding cetuximab to capecitabine plus oxaliplatin (XELOX) in first-line treatment of metastatic colorectal cancer: a randomized phase II trial of the Swiss Group for Clinical Cancer Research SAKK. Ann Oncol 2008, 19(7):1288-1292.

31. Arnold D, Hohler T, Dittrich C, Lordick F, Seufferlein T, Riemann J, Woll E, Herrmann T, Zubel A, Schmoll HJ: Cetuximab in combination with weekly 5-fluorouracil/folinic acid and oxaliplatin (FUFOX) in untreated patients with advanced colorectal cancer: a phase Ib/ll study of the AIO GI Group. Ann Oncol 2008, 19(8):1442-1449.

32. Cartwright T, Kuefler P, Cohn A, Hyman W, Berger M, Richards D, Vukelja S, Nugent JE, Ruxer RL Jr, Boehm KA, et al: Results of a phase II trial of cetuximab plus capecitabine/irinotecan as first-line therapy for patients with advanced and/or metastatic colorectal cancer. Clin Colorectal Cancer 2008, 7(6):390-397.

33. Van Cutsem E, Kohne $\mathrm{CH}$, Hitre E, Zaluski J, Chang Chien $\mathrm{CR}$, Makhson $\mathrm{A}$, D'Haens G, Pinter T, Lim R, Bodoky G, et al: Cetuximab and chemotherapy as initial treatment for metastatic colorectal cancer. N Engl J Med 2009, 360(14):1408-1417.

34. Bokemeyer C, Bondarenko I, Makhson A, Hartmann JT, Aparicio J, de Braud F, Donea S, Ludwig H, Schuch G, Stroh C, et al: Fluorouracil, leucovorin, and oxaliplatin with and without cetuximab in the first-line treatment of metastatic colorectal cancer. J Clin Oncol 2009, 27(5):663-671.

35. Tol J, Koopman M, Cats A, Rodenburg CJ, Creemers GJ, Schrama JG, Erdkamp FL, Vos AH, van Groeningen CJ, Sinnige HA, et al: Chemotherapy, bevacizumab, and cetuximab in metastatic colorectal cancer. $N$ Engl $J$ Med 2009, 360(6):563-572.

36. Maughan TS, Adams RA, Smith CG, Meade AM, Seymour MT, Wilson RH, Idziaszczyk S, Harris R, Fisher D, Kenny SL, et al: Addition of cetuximab to oxaliplatin-based first-line combination chemotherapy for treatment of advanced colorectal cancer: results of the randomised phase 3 MRC COIN trial. Lancet 2011, 377(9783):2103-2114.

37. Tveit KM, Guren T, Glimelius B, Pfeiffer P, Sorbye H, Pyrhonen S, Sigurdsson F, Kure E, Ikdahl T, Skovlund E, et al: Phase III trial of cetuximab with continuous or intermittent fluorouracil, leucovorin, and oxaliplatin (Nordic FLOX) versus FLOX alone in first-line treatment of metastatic colorectal cancer: the NORDIC-VII study. J Clin Oncol 2012, 30(15):1755-1762.

38. Saltz LB, Meropol NJ, Loehrer PJ Sr, Needle MN, Kopit J, Mayer RJ: Phase II trial of cetuximab in patients with refractory colorectal cancer that expresses the epidermal growth factor receptor. J Clin Oncol 2004, 22(7):1201-1208

39. Jonker DJ, O'Callaghan CJ, Karapetis CS, Zalcberg JR, Tu D, Au HJ, Berry SR, Krahn M, Price T, Simes RJ, et al: Cetuximab for the treatment of colorectal cancer. N Engl J Med 2007, 357(20):2040-2048

40. Cunningham D, Humblet $Y$, Siena S, Khayat D, Bleiberg H, Santoro A, Bets D, Mueser M, Harstrick A, Verslype C, et al: Cetuximab monotherapy and cetuximab plus irinotecan in irinotecan-refractory metastatic colorectal cancer. N Engl J Med 2004, 351(4):337-345.

41. Van Cutsem E, Kohne CH, Lang I, Folprecht G, Nowacki MP, Cascinu S, Shchepotin I, Maurel J, Cunningham D, Tejpar S, et al: Cetuximab plus irinotecan, fluorouracil, and leucovorin as first-line treatment for metastatic colorectal cancer: updated analysis of overall survival according to tumor KRAS and BRAF mutation status. J Clin Oncol 2011, 29(15):2011-2019.

42. Bokemeyer C, Cutsem EV, Rougier P, Ciardiello F, Heeger S, Schlichting M, Celik I, Kohne CH: Addition of cetuximab to chemotherapy as first-line treatment for KRAS wild-type metastatic colorectal cancer: pooled analysis of the CRYSTAL and OPUS randomised clinical trials. Eur I Cancer 2012, 48(10):1466-1475.

43. Peeters M, Price TJ, Cervantes A, Sobrero AF, Ducreux M, Hotko Y, Andre T, Chan E, Lordick F, Punt CJ, et al: Randomized phase III study of panitumumab with fluorouracil, leucovorin, and irinotecan (FOLFIRI) compared with FOLFIRI alone as second-line treatment in patients with metastatic colorectal cancer. J Clin Oncol 2010, 28(31):4706-4713.

44. Franko J, Shi Q, Goldman CD, Pockaj BA, Nelson GD, Goldberg RM, Pitot HC, Grothey A, Alberts SR, Sargent DJ: Treatment of colorectal peritoneal carcinomatosis with systemic chemotherapy: a pooled analysis of north central cancer treatment group phase III trials N9741 and N9841. J Clin Oncol 2012, 30(3):263-267.

45. Klaver YL, Simkens LH, Lemmens VE, Koopman M, Teerenstra S, Bleichrodt RP, de Hingh IH, Punt CJ: Outcomes of colorectal cancer patients with peritoneal carcinomatosis treated with chemotherapy with and without 
targeted therapy. Eur J Surg Oncol 2012, 38(7):617-623.

46. Elias D, Lefevre JH, Chevalier J, Brouquet A, Marchal F, Classe JM, Ferron G, Guilloit JM, Meeus P, Goere D, et al: Complete cytoreductive surgery plus intraperitoneal chemohyperthermia with oxaliplatin for peritoneal carcinomatosis of colorectal origin. J Clin Oncol 2009, 27(5):681-685.

47. Lee DH, Oh SY, Lee YR, Huh SJ, Yoon HH, Kim SH, Lee S, Lee JH, Kim Y, Kim HJ, et al: A phase II study of modified FOLFOX4 for colorectal cancer patients with Peritoneal Carcinomatosis. Cancer Res Treat 2011, 43(4):225-230.

48. Sugarbaker PH, Cunliffe WJ, Belliveau J, de Bruijn EA, Graves T, Mullins RE, Schlag P: Rationale for integrating early postoperative intraperitoneal chemotherapy into the surgical treatment of gastrointestinal cancer. Semin Oncol 1989, 16(4 Suppl 6):83-97.

49. Sugarbaker PH: New standard of care for appendiceal epithelial neoplasms and pseudomyxoma peritonei syndrome? Lancet Oncol 2006, 7(1):69-76.

50. Dedrick RL: Theoretical and experimental bases of intraperitoneal chemotherapy. Semin Oncol 1985, 12(3 Suppl 4):1-6

51. Flessner MF, Dedrick RL, Schultz JS: A distributed model of peritonealplasma transport: analysis of experimental data in the rat. Am J Physiol 1985, 248(3 Pt 2):F413-F424.

52. Speyer $\mathrm{L}$, Collins JM, Dedrick RL, Brennan MF, Buckpitt AR, Londer H, DeVita $V T$ Jr, Myers CE: Phase I and pharmacological studies of 5-fluorouracil administered intraperitoneally. Cancer Res 1980, 40(3):567-572.

53. Sugarbaker PH, Stuart OA, Carmignani CP: Pharmacokinetic changes induced by the volume of chemotherapy solution in patients treated with hyperthermic intraperitoneal mitomycin C. Cancer Chemother Pharmacol 2006, 57(5):703-708.

54. Elias D, Matsuhisa T, Sideris L, Liberale G, Drouard-Troalen L, Raynard B, Pocard M, Puizillou JM, Billard V, Bourget $P$, et al: Heated intra-operative intraperitoneal oxaliplatin plus irinotecan after complete resection of peritoneal carcinomatosis: pharmacokinetics, tissue distribution and tolerance. Ann Oncol 2004, 15(10):1558-1565.

55. Elias D, Bonnay M, Puizillou JM, Antoun S, Demirdjian S, El OA, Pignon JP, Drouard-Troalen L, Ouellet JF, Ducreux M: Heated intra-operative intraperitoneal oxaliplatin after complete resection of peritoneal carcinomatosis: pharmacokinetics and tissue distribution. Ann Oncol 2002, 13(2):267-272.

56. Gonzalez-Moreno S, Gonzalez-Bayon LA, Ortega-Perez G: Hyperthermic intraperitoneal chemotherapy: Rationale and technique. World $J$ Gastrointest Oncol 2010, 2(2):68-75.

57. Sugarbaker PH: Laboratory and clinical basis for hyperthermia as a component of intracavitary chemotherapy. Int J Hyperthermia 2007, 23(5):431-442

58. Issels RD: Hyperthermia adds to chemotherapy. Eur J Cancer 2008, 44(17):2546-2554.

59. Verwaal VJ, Bruin S, Boot H, van Slooten G, van Tinteren H: 8-year follow-up of randomized trial: cytoreduction and hyperthermic intraperitoneal chemotherapy versus systemic chemotherapy in patients with peritoneal carcinomatosis of colorectal cancer. Ann Surg Oncol 2008, 15(9):2426-2432.

60. Verwaal VJ, van Ruth S, de Bree E, van Sloothen GW, van Tinteren H, Boot H, Zoetmulder FA: Randomized trial of cytoreduction and hyperthermic intraperitoneal chemotherapy versus systemic chemotherapy and palliative surgery in patients with peritoneal carcinomatosis of colorectal cancer. J Clin Oncol 2003, 21(20):3737-3743.

61. Elias D, Delperro JR, Sideris L, Benhamou E, Pocard M, Baton O, Giovannini $M$, Lasser P: Treatment of peritoneal carcinomatosis from colorectal cancer: impact of complete cytoreductive surgery and difficulties in conducting randomized trials. Ann Surg Oncol 2004, 11(5):518-521.

62. Mahteme H, Hansson J, Berglund A, Pahlman L, Glimelius B, Nygren P, Graf W: Improved survival in patients with peritoneal metastases from colorectal cancer: a preliminary study. Br J Cancer 2004, 90(2):403-407.

63. Glehen O, Kwiatkowski F, Sugarbaker PH, Elias D, Levine EA, De Simone M, Barone R, Yonemura Y, Cavaliere F, Quenet F, et al: Cytoreductive surgery combined with perioperative intraperitoneal chemotherapy for the management of peritoneal carcinomatosis from colorectal cancer: a multi-institutional study. J Clin Oncol 2004, 22(16):3284-3292.

64. Glockzin G, Ghali N, Lang SA, Agha A, Schlitt HJ, Piso P: Peritoneal carcinomatosis. Surgical treatment, including hyperthermic intraperitoneal chemotherapy. Chirurg 2007, 78(12):1100-1110.

65. Hompes D, D'Hoore A, Van Cutsem E, Fieuws S, Ceelen W, Peeters M, Van der Speeten K, Bertrand C, Legendre H, Kerger J: The treatment of peritoneal carcinomatosis of colorectal cancer with complete cytoreductive surgery and hyperthermic intraperitoneal peroperative chemotherapy (HIPEC) with oxaliplatin: a Belgian multicentre prospective phase II clinical study. Ann Surg Oncol 2012, 19(7):2186-2194.

66. Pilati P, Mocellin S, Rossi CR, Foletto M, Campana L, Nitti D, Lise M: Cytoreductive surgery combined with hyperthermic intraperitoneal intraoperative chemotherapy for peritoneal carcinomatosis arising from colon adenocarcinoma. Ann Surg Oncol 2003, 10(5):508-513.

67. Glehen O, Cotte E, Schreiber V, Sayag-Beaujard AC, Vignal J, Gilly FN: Intraperitoneal chemohyperthermia and attempted cytoreductive surgery in patients with peritoneal carcinomatosis of colorectal origin. Br J Surg 2004, 91(6):747-754.

68. Shen P, Hawksworth J, Lovato J, Loggie BW, Geisinger KR, Fleming RA, Levine EA: Cytoreductive surgery and intraperitoneal hyperthermic chemotherapy with mitomycin C for peritoneal carcinomatosis from nonappendiceal colorectal carcinoma. Ann Surg Oncol 2004, 11(2):178-186.

69. Quenet F, Claus C, Roca L, Gauthey A, Essissen M, Ychou M, Rouanet P, SaintAubert B: Complete cytoreductive surgery and hyperthermic intraperitoneal chemotherapy for peritoneal carcinomatosis from digestive tract cancer-New management with oxaliplatin plus irinotecan: a feasibility study in 37 patients. J Clin Oncol 2008, 26. suppl; abstr 4084

70. Glehen O, Gilly FN: Quantitative prognostic indicators of peritoneal surface malignancy: carcinomatosis, sarcomatosis, and peritoneal mesothelioma. Surg Oncol Clin N Am 2003, 12(3):649-671.

71. Jacquet $\mathrm{P}$, Sugarbaker $\mathrm{PH}$ : Clinical research methodologies in diagnosis and staging of patients with peritoneal carcinomatosis. Cancer Treat Res 1996, 82:359-374.

72. Glockzin G, Schlitt HJ, Piso P: Peritoneal carcinomatosis: patients selection, perioperative complications and quality of life related to cytoreductive surgery and hyperthermic intraperitoneal chemotherapy. World I Surg Oncol 2009, 7(1):5.

73. Eisenhauer EA, Therasse P, Bogaerts J, Schwartz LH, Sargent D, Ford R, Dancey J, Arbuck S, Gwyther S, Mooney M, et al: New response evaluation criteria in solid tumours: revised RECIST guideline (version 1.1). Eur J Cancer 2009, 45(2):228-247.

74. Younan R, Kusamura S, Baratti D, Cloutier AS, Deraco M: Morbidity, toxicity, and mortality classification systems in the local regional treatment of peritoneal surface malignancy. J Surg Oncol 2008, 98(4):253-257.

75. Fayers PM: Interpreting quality of life data: population-based reference data for the EORTC QLQ-C30. Eur J Cancer 2001, 37(11):1331-1334.

76. Schwarz R, Hinz A: Reference data for the quality of life questionnaire EORTC QLQ-C30 in the general German population. Eur J Cancer 2001, 37(11):1345-1351.

77. Koller M, Lorenz W: Quality of life: a deconstruction for clinicians. J R Soc Med 2002, 95(10):481-488.

78. Dworak $\mathrm{O}$, Keilholz $\mathrm{L}$, Hoffmann A: Pathological features of rectal cancer after preoperative radiochemotherapy. Int J Colorectal Dis 1997, 12(1):19-23.

79. Rubinstein LV, Korn EL, Freidlin B, Hunsberger S, Ivy SP, Smith MA: Design issues of randomized phase II trials and a proposal for phase II screening trials. J Clin Oncol 2005, 23(28):7199-7206.

80. Lawless JF: Statistical Models and Methods for Lifetime Data (Wiley Series in Probability \& Mathematical Statistics). New York: John Wiley \& Sons; 1982.

81. Glockzin G, von Breitenbuch P, Schlitt HJ, Piso P: Treatment-related morbidity and toxicity of CRS and oxaliplatin-based HIPEC compared to a mitomycin and doxorubicin-based HIPEC protocol in patients with peritoneal carcinomatosis: A matched-pair analysis. J Surg Oncol 2012, 12(1):356.

doi:10.1186/1471-2407-13-67

Cite this article as: Glockzin et al:: A prospective multicenter phase II study evaluating multimodality treatment of patients with peritoneal carcinomatosis arising from appendiceal and colorectal cancer: the COMBATAC trial BMC Cancer 2013 13:67. 